\title{
Journal of Hematology \& Oncology reviewer acknowledgement 2015
}

\author{
Delong Liu
}

\section{Contributing reviewers}

The Editor-in-Chief of Journal of Hematology \& Oncology would like to thank all our reviewers who have contributed to the journal in Volume 8 (2015).

Nacer Abrouk

United States of America

Akintunde Akinleye

United States of America

Yoshimitsu Akiyama

Japan

Ugo Ala

Italy

A Alousi

United States of America

Xiuli An

United States of America

Ephraim Ansa-Addo

United States of America

Emmanuel Antonarakis

United States of America

Dorina Avram

United States of America

Veronika Bachanova

United States of America

Veronica Balatti

United States of America

Srinivasa Rao Bandi

United States of America

Frederic Baron

Belgium

\section{Rodolphe Barrangou}

United States of America

Giuseppe Basso

Italy

Ioana Berindan-Neagoe

Romania

Paolo Bernasconi

Kazakhstan

Jin-Song Bian

Singapore

Andrea Biondi

Italy

Cf Boerkoel

Canada

Arnold Bolomsky

Austria

Earle Burgess

United States of America

Hong Chang

Canada

Ying-Jun Chang

China

Huan Chen

China

Suning Chen

China

\author{
Guoan Chen \\ United States of America
}

M Chen

United States of America

Vicky Ping Chen

United States of America

Tao Cheng

China

Hong Cheng

United States of America

Seok-Goo Cho

South Korea

Eun Young Choi

Korea, South

Shih-Sung Chuang

Taiwan

Mei-Zhen Cui

United States of America

Silvia Deaglio

Italy

Jiusheng Deng

United States of America

Fei Duan

United States of America

William Durante

United States of America

Correspondence: jhoonline@biomedcentral.com

Department of Medicine, New York Medical College and Westchester

Medical Center, New York, NY, USA 
Xiucheng Fan

United States of America

Qipeng Fan

United States of America

Zhichao Fan

United States of America

Mark Feinberg

United States of America

Michael Flister

United States of America

Karlheinz Friedrich

Germany

Yanfang Fu

United States of America

Seiji Fukuda

Japan

Claudia Fumarola

Italy

Sara Galimberti

Italy

S Gallego

Spain

Gregory Gan

United States of America

Joan Gil

Spain

Alois Gratwohl

United States of America

Charlotte Grootaert

Belgium

Aihua Gu

China

Ming Guan

China

Christina Halsey

United Kingdom

\section{Lina Han}

United States of America

Bo Han

United States of America

Donald Harvey

United States of America

Lizhi He

United States of America
Yukai He

United States of America

Mark Heaney

United States of America

Cyrus Hedvat

United States of America

Tom Hobman

Canada

Jian Hou

China

George Hsiao

Taiwan

Wenming Hsu

United States of America

Chung-Tsen Hsueh

United States of America

Qinglong $\mathrm{Hu}$

United States of America

Shimin Hu

United States of America

Wenwei $\mathrm{Hu}$

United States of America

He Huang

China

Xiaojun Huang

China

Huang Huang

United States of America

Suming Huang

United States of America

Tiangui Huang

United States of America

Tsong-Long Hwang

Taiwan

William Hwang

Singapore

Toshihiko Imamura

Japan

Benjamin Jackson

United States of America

Gaetan Jego

France

Yuxia Jia

United States of America
Wei Jiang

United States of America

Dan Jones

United States of America

Celina Juliano

United States of America

Hirokazu Kanegane

Japan

Khosrow Kashfi

United States of America

Xiao Yan Ke

China

Fadi Khasawneh

United States of America

Joseph Khoury

United States of America

Edward Kim

United States of America

Seok Jin Kim

South Korea

Won Seog Kim

South Korea

M Kitagawa

Japan

Maria Kleppe

United States of America

Ilona Kovalszky

Hungary

Dieter Kube

Germany

Y Lai

China

Gurpreet Lamba

United States of America

Jungwoo Lee

United States of America

Mong-Hong Lee

United States of America

Robert Lerner

United States of America

Chi-Kwong Li

Hong Kong

Jin Li

China 
Ji-Liang Li

United Kingdom

Peng Li

China

Yong $\mathbf{L i}$

United States of America

Qiao Li

China

Shaoguang Li

United States of America

Tianhong Li

China

Guangfu Li

United States of America

Shaoguang Li

United States of America

Yangqiu Li

China

Zhenyu Li

United States of America

Zihai Li

United States of America

Felix Lichtenegger

Germany

Edward Lin

United States of America

Jianqing Lin

United States of America

Hongtao Liu

United States of America

Jianguo Liu

United States of America

Qi-Fa Liu

China

Zach Liu

United States of America

Sanam Loghavi

United States of America

Min Lu

China

Harry Lybeck

United States of America

Manisha Madkaikar

United States of America
Atsushi Manabe

Japan

Luigi Marcheselli

Italy

Philip Mason

United States of America

Rohit Mathur

United States of America

Sebastien Maury

France

Helmout Modjtahedi

United Kingdom

S Montes-Moreno

Spain

Hideki Muramatsu

Japan

Hiroshi Nakano

Japan

Heyu Ni

Canada

Maria Odero

Spain

Seiichi Okabe

Japan

Dennis O'malley

United States of America

Thomas Pabst

Switzerland

Minggui Pan

United States of America

Gyorgy Panyi

Hungary

Simrit Parmar

United States of America

Luiz O. Penalva

United States of America

Piergiorgio Petroni

Italy

Simon Powis

United Kingdom

B Psaila

United Kingdom

Zhenggang Ren

China
Ruibao Ren

United States of America

Uwe Reusch

Germany

Jia Ruan

United States of America

Ko Sato

Japan

Martin Sauvageau

United States of America

Tobias Schatton

United States of America

Ig Schmidt-Wolf

Germany

Karen Seiter

United States of America

Lohith Shakaladevanapura

United States of America

He Shen

United States of America

Yuankai Shi

China

Sy Shieh

United States of America

Yongqian Shu

China

Irma Rosa Slavutsky

Argentina

Jürgen Sonnemann

Germany

Carmen Stanganelli

Argentina

Gp Studzinski

United States of America

Vivek Subbiah

United States of America

Marion Subklewe

Germany

M Suenaga

Japan

Hui-Chuan Sun

China

Daniel Sze

Australia 


\author{
Andrew Tan \\ Singapore \\ Guilin Tang \\ United States of America \\ Jianguo Tao \\ United States of America \\ Jessica Thaxton \\ United States of America \\ Erming Tian \\ United States of America \\ Raoul Tibes \\ United States of America \\ Ramon Tiu \\ United States of America \\ K Tokoyoda \\ Germany \\ Che-Kai Tsao \\ United States of America \\ Panagiotis Tsirigotis \\ Israel \\ Jeffrey Tyner \\ United States of America \\ Ikuyo Ueda \\ Japan
}

Diamantina Vasilatou

Greece

Amit Verma

United States of America

Massimiliano Visocchi

United States of America

Phuong Vo

United States of America

Sinisa Volarevic

Croatia

Jing Wang

United States of America

Jiyi Wang

United States of America

Sa Wang

United States of America

Qinhong Wang

United States of America

Wei Wang

United States of America
Xiaoxiao Wang

United States of America

Britta Will

United States of America

Petter Woll

United States of America

Justin Wong

Australia

John Wrangle

United States of America

Kongming Wu

China

Shenhong Wu

United States of America

Yi-Long Wu

China

Jingjing $\mathbf{W u}$

China

Lijun Xia

United States of America

Zhijian Xiao

China

Yu-Quan Xiong

China

Binghe Xu

China

Dazhong Xu

United States of America

Ruihua Xu

China

Ozlem Yalcin

United States of America

Masayuki Yamamoto

Japan

Shuo Yang

United States of America

Naohiro Yano

United States of America

Jeffrey Ye

United States of America

Go Yoshida

Japan

Scott Younger

United States of America
Evan Yu

United States of America

Min Yu

United States of America

Jianda Yuan

United States of America

Zihua Zeng

United States of America

Hua Zhang

China

Lanjing Zhang

United States of America

Chengcheng Zhang

United States of America

Xi Zhang

United States of America

Xu Zhang

China

Yue Zhang

United States of America

Jing Zhang

United States of America

Ti Zhang

China

Wei-Li Zhao

China

Guo-Guang Zheng

China

Xiaoyong Zheng

United States of America

Huyong Zheng

China

Wenxin Zheng

United States of America

Xiaoyong Zheng

United States of America

Yong Zhou

China

Niklas Zojer

Austria 